years). The most common clinical manifestations at onset were fatigue (78.4\%), Anaemia (72.2\%), polyarthralgia (66.2\%), photosensitivity (61.1\%), low grade fever (56.6\%) and myalgias (52.1\%). Renal involvement was seen in 56.3\% subjects. ANA by immunofluorescence was positive in $100 \%$ and most common pattern was speckled (62.1\%). Immunoblot assay for sub autoantibodies showed Anti DsDNA (56.7\%), Antinucleosome (25.6\%), antihistones (28.4\%), anti SmD1 (28.2\%), anti Ro52 Kd (58.4\%) and Anti Ro $60 \mathrm{kd}(52.2 \%)$. A total of 08 patients died during follow up and most common cause was sepsis with underlying renal involvement.

Conclusions This retrospective study on a large cohort of SLE patients from India shows significant difference in clinical manifestations and autoantibody profile in Asians as compared to Caucasians.

\section{CORRELATION OF HISTOPATHOLOGY WITH CLINICAL PARAMETERS IN LUPUS NEPHRITIS AMONG FILIPINOS}

E Venegas*, A de Asis- Fabila, S Navarra. University of Santo Tomas Hospital, Rheumatology, Manila, Philippines

\subsection{6/lupus-2017-000215.240}

Background and aims In Philippine setting, management of lupus nephritis (LN) is primarily driven by clinical parameters more than kidney biopsy because of limited resources. This paper describes clinico-pathologic correlations in a cohort of Filipino patients with LN.

Methods Study population included LN patients who underwent kidney biopsy at University of Santo Tomas (UST) Hospital, Manila, Philippines from 2005 to 2015.Pathologic diagnoses utilised International Society of Nephrology/Renal Pathology Society (ISN/RPS) 2003 classification, including activity and chronicity indices. Correlations of histopathologic classification with demographic and systemic lupus erythematosus (SLE) characteristics were performed using linear and multinomial regression analysis.

Results Included were $101 \mathrm{LN}$ patients (94 females, 72 adults) with mean age $25.2 \pm 11.5$ (9-61) at SLE diagnosis and 3.08 $\pm 6.02(<1-12)$ years disease duration from SLE diagnosis to biopsy. Most common ISN/RPS classification was Class IV in 57 (56.4\%) patients and Class III in 33 (32.7\%). Average activity index was $6.64 \pm 2.22(0-12)$, chronicity index, 3.54 \pm 2.02 (0-9); Class IV and V correlated with higher activity index scores, $\mathrm{p}=0.001$. Mean uPCR was $2.61+1.44(0.03-$ $7.43) \mathrm{mg} / \mathrm{mg}$ with highest uPCR in Class IV, followed by classes III and V. Mean estimated glomerular filtration rate (eGFR) was 63.02 $\pm 34.25(9-139) \mathrm{mL} / \mathrm{min}$, with inverse correlation between eGFR and histologic activity, $p=0.003$. Extra renal manifestations included arthritis (61\%), malar rash (59\%) and photosensitivity (50\%), with mean SLEDAI score of $11.07 \pm 3.78$; these did not correlate with histologic indices. Conclusions This study shows good correlation of clinical renal parameters with histopathology, supporting the rationale of current Philippine practice to perform kidney biopsies as clinically indicated rather than routinely.

\section{ROLE OF SEROLOGY IN DIAGNOSIS OF EARLY SLE AMONG FILIPINOS}

'L. Zamora*. 'University of Santo Tomas Hospital, Medicine Section of Rheumatology, Manila, Philippines

\subsection{6/lupus-2017-000215.241}

Background and aims Variable evolution of manifestations and reliance on serology for definitive SLE classification in early systemic lupus erythematosus (SLE) can challenge shared patient-physician management decisions and strain limited resources. This study aimed to determine which clinical manifestation/s require additional serology to formally classify early SLE patients.

Methods Clinical and serologic manifestations of patients with early SLE diagnosed $<1$ year from symptom onset at Lupus Clinics of University of Santo Tomas (UST) Hospital, Manila, Philippines from January 2014 to December 2015 were analysed. Minimum laboratory tests included complete blood count (CBC), urinalysis and anti-nuclear antibody (ANA). Clinical manifestations were based on the 2012 SLICC criteria.

Results 79 patients (78 females) had mean age at SLE diagnosis of $31.95+10.5$ years (range 18-53), mean disease duration 5.66+5.41 months (range 0.23-12), all patients were ANA positive. Most common clinical manifestations were alopecia, acute cutaneous lupus rash (malar and photosensitive rash), arthritis and nephritis. Sixty-five patients (82.3\%) fulfilled at least 3 clinical criteria for SLE. Fourteen patients required additional serology to complete classification criteria: 12 patients had only 2 clinical criteria including mucocutaneous $(n=11)$, arthritis $(n=6)$ and nephritis $(n=4)$; the other 2 patients had only 1 clinical criterion each as thrombocytopenia or nephritis.

Conclusions In this early SLE cohort, mucocutaneous and musculoskeletal were the most common presenting manifestations. Additional serology was more often required in those with "asymptomatic" features of nephritis and thrombocytopenia when other clinical features are absent - reinforcing the value of $\mathrm{CBC}$ and urinalysis in early SLE.

\section{LONG-TERM PROGNOSIS OF PATIENTS WITH SYSTEMIC LUPUS ERYTHEMATOSUS-ASSOCIATED PULMONARY ARTERIAL HYPERTENSION: CSTAR-PAH COHORT STUDY}

J Zhao*, J Qian, W Qian, M Li, X Zeng. Peking Union Medical College Hospital, Rheumatology, beijing, China

\subsection{6/lupus-2017-000215.242}

Background and aims Systemic lupus erythematosus (SLE)-associated pulmonary arterial hypertension (PAH) is common in Asian countries, and the clinical outcome of patients with SLE-associated PAH is dramatically impaired. This study aimed to identify the long-term clinical outcomes and prognostic factors of patients with SLE-associated PAH confirmed by right heart catheterization (RHC).

Methods A multicenter cohort of SLE-associated PAH was established. Baseline and follow-up records were collected. The primary endpoint was death from any cause. The secondary experimental end point was treatment goal achievement (TGA).

Results Among the 310 patients enrolled from $14 \mathrm{PAH}$ centres, 282 patients with confirmed mortality statuses were included in the survival analysis, 263 patients with complete 
follow-up data were included in the TGA study. The median follow-up was 24.0 months. The 1-, 3- and 5 year survival rates were $92.1 \%, 84.8 \%$ and $72.9 \%$, respectively. The 1-, 3and 5 year TGA rates were $31.5 \%, 53.6 \%$ and $62.7 \%$, respectively. Serositis $(\mathrm{HR}=1.94,95 \% \mathrm{CI}: 1.26-3.00, \mathrm{p}=0.003)$, $6 \mathrm{MWD}>380 \mathrm{~m} \quad(\mathrm{HR}=1.95,95 \% \mathrm{CI}: 1.14-3.31, \mathrm{p}=0.014)$ and $\quad \mathrm{CI} \geq 2.5 \mathrm{~L} / \mathrm{min} \times \mathrm{m}^{2} \quad(\mathrm{HR}=1.92, \quad 95 \% \mathrm{CI}: \quad 1.16-3.19$, $\mathrm{p}=0.012$ ) were identified as independent prognostic factors of TGA.TGA within 5 years was identified as a factor associated with survival in patients with SLE-associated PAH.

Conclusions TGA was associated with the long-term survival, which supports and provides evidence to the treat-to-target strategy in SLE-associated PAH. Early diagnosis, intervention and heart function preservation are priorities for better longterm outcomes. PAH patients with high SLE activity may benefit from immunosuppressive therapy.

A

B

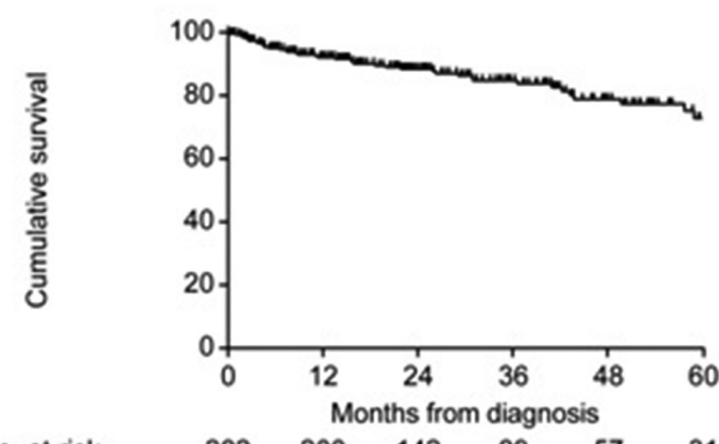

No. at risk

282200

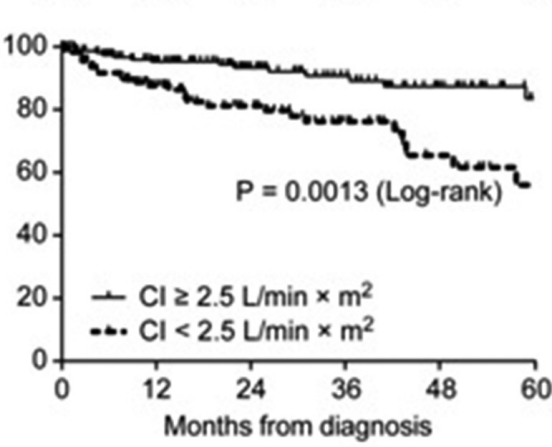

No. at risk

$\begin{array}{lllllll}\mathrm{Cl} \geq 2.5 \mathrm{~L} / \mathrm{min} \times \mathrm{m}^{2} & 166 & 123 & 87 & 58 & 40 & 21 \\ \mathrm{Cl}<2.5 \mathrm{~L} / \mathrm{min} \times \mathrm{m}^{2} & 116 & 78 & 56 & 32 & 18 & 11\end{array}$

C

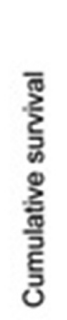

No. at risk RAP $\leq 10 \mathrm{mmHg}$ RAP $>10 \mathrm{mmHg}$

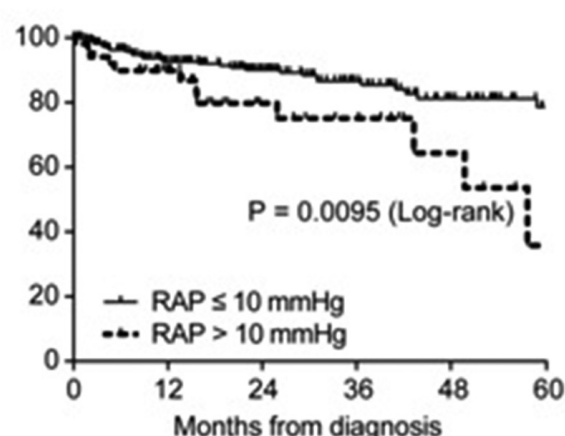

Months from diagnosis

$\begin{array}{cccccc}227 & 168 & 125 & 76 & 51 & 29 \\ 55 & 33 & 18 & 14 & 7 & 3\end{array}$

\section{3}

THE PIVOTAL ROLE OF INTENSIVE IMMUNOSUPPRESSIVE THERAPY IN THE MANAGEMENT OF SYSTEMIC LUPUS ERYTHEMATOSUS ASSOCIATED WITH PULMONARY ARTERIAL HYPERTENSION

'J Zhao*, 'Q Wang, 'J Qian, ${ }^{2} Z$ Tian, ${ }^{2} Y$ Liu, ${ }^{1} \mathrm{M}$ Li, ${ }^{1} X$ Zeng. ${ }^{1}$ Peking Union Medical College Hospital, Rheumatology, beijing, China; ${ }^{2}$ Peking Union Medical College Hospital, Cardiology, beijing, China

\subsection{6/lupus-2017-000215.243}

Background and aims Immune and inflammatory mechanisms could play a significant role in pulmonary arterial hypertension $(\mathrm{PAH})$ genesis and progression, especially in patients with systemic lupus erythematosus (SLE). Immunosuppressive therapy should be better evaluated in this setting. We reviewed the clinical outcomes of intensive immunosuppressive therapy with or without target therapy in SLE associated P

Methods This single-centre cohort study enrolled 126 consecutive patients with SLE-PAH who visited our referral centre in China between May 2006 and December 2015. Baseline demographics, clinical features, laboratory results, haemodynamic assessments and management were analysed. Kaplan-Meier curves and Cox proportional hazards regression analysis were used toevaluate the role of intensive immunosuppressive therapy.

Results ALL patients received intensive immunosuppressive therapy including combination of high-dose glucocorticosteroids and first-line immunosuppressants, such as cyclophosphamide, mycophenolate and calcineurin Inhibitors. Eighty-two $(65.1 \%)$ patients received target therapy at baseline. Survival analysis indicated that responders had a better survival than nonresponders in both with and without target therapy group (figure 1). Patients with a shorter SLE disease duration $(p=0.009)$ and better baseline pulmonary hemodynamics (mean pulmonary arterial pressure, pulmonary vascular resistance and Cardiac index, $\mathrm{p}<0.001$ ) were more likely to benefit from immunosuppressive therapy (table 1).

Conclusions Intensive immunosuppressive therapy markedly improved the long-term outcomes of SLE patients with PAH, especially in the early stage of PAH.

\section{LONG-TERM PROGNOSIS AND PREDICTING FACTORS OF CHINESE PATIENTS WITH SYSTEMIC LUPUS ERYTHEMATOSUS: A MULTI-CENTRECENTER COHORT STUDY FROM CSTAR REGISTRY}

IJ Zhao*, 'Z Wang, ${ }^{2} Y$ Wang, ${ }^{1} \mathrm{Q}$ Wang, ${ }^{1} \mathrm{M}$ Li, ${ }^{1} \mathrm{X}$ Zeng. ${ }^{1}$ Peking Union Medical College Hospital, Rheumatology, beijing, China; ${ }^{2}$ School of Basic Medicine Peking Union Medical College and Institute of Basic Medical Sciences Chinese Academy of Medical Sciences, Department of Epidemiology and Biostatistics, beijing, China

\subsection{6/lupus-2017-000215.244}

Background and aims To investigate the long-term outcomes, both mortality and damage, and predict factors of patients with systemic lupus erythematosus (SLE) in the CSTAR (Chinese SLE Treatment and Research group) registry cohort.

Methods Patients were enrolled from April 2009 to February 2010. They were followed up at clinic and were telephone interviewed at the endpoint. Demographic data, clinical manifestations, activity, damage scores, and medications were collected. Survival rates were studied by Kaplan-Meier method, 